erscheinungen ausübt, so lassen sich hieraus Unterschiede in der Wirkungs. weise beider genannter Reaktionsarten aufbauen. Dieselben müssen je nach Konzentration, Temperatur, Zeitdauer, und je nach der chemischen, nicht zuletzt aber auch der phys kalischeir Art des Reaktionsobjekts und des
Dispersitätsgrades * seiner Komponenten verschiedenartig ausfallen; besondere Beachtung verdient hierbei die Rolle des Magnesiumkarbonats als dauernder Träger mäBiger Alkalität, besonders in der Hitze.

\section{Miinchen.}

Betriebslaboratorium der Spatenbrauerei.

\title{
Studien über Schutzkolloide. Vierte Reihe: Semen Lini als Schutzkolloid.
}

\section{Mitteilung: Allgemeine kolloidehemische Untersuchungen über den Leinsamenschleim.}

\author{
Von A. Gutbier, J. Huber und E. Kuhn t. \\ (Eingeg. an 24. Mkrz 1816.) \\ (Mitteilang aus dem Laboratorium für Elektrochemie und Technische Chemie det Technischen Hochschule \\ Stuttgart.)
}

Die Leinpflanze, aus deren Samen wir ebenfalls einen für kolloidchemische Zwecke brauchbaren Schleim gewonnen haben, ist vielleicht in den Kaukasusländem einheimisch ${ }^{1}$ ), ihre Kultur ist aber schon seit langer Zeit weit verbreitet. Der Samen ist eiförmig, flach und scharfrandig, an einem Pole gerundet, am anderen, dem Mikropylarende, etwas eingedrückt und benabelt. Die Samenschale ist braun oder gelblich, glatt, spröde, umschliebt in einem dünnen Endosperm den Embryo mit zwei dicken, flachen Kotyledonen und dem dicken Würzelchen, und weist folgende Schichten auf: a) die schleimgebende Epidermis mit Kutikula, gebildet aus großen, nahezu würfeligen oder radial verlängerten, kurz prismatischen Zellen. von eckigem Umrisse des Querschnitts; an der inneren AuBenwand sind dicke Schleimlamellen als Membranverdickung aufgelagert; b) eine einfache oder doppelte Lage dünnwandiger, polyedrischer Zellen; c) eine Lage stark verdickter, poröser, kurzer Fasern; d) eine Nährschicht. deren Zellen mit den Fasern der vorigen Schicht gekreuzt sind; e) die, aus im Längschnitte fast isodiametrischen oder quadratischen Zellen bestehende Pigmentschicht, die der Schale des sogenannten ,hellen indischen Leinsamens" bekanntlich fehlt, und bei der die Zellwände'sehr fein getüpfelt sind, während die Zellen selbst einen braunen Inhal haben; $f$ ) eine dünne Zone obliterierten Gewebes.

Die Literatur über Leinsamen ist verhältnismäBig umfangreich, doch beschäftigen sich die meisten Arbeiten mit dem Gehalte der Samen an Oel, der für unsere Betrachtungen nicht

i) Vgl. Hager's ,Handbuch der Pharmazeutischen Praxis", 3. Aufi. 2, 297 (1903 . weiter $z u$ berücksichtigen ist. Von den anderen Untersuchungen, soweit sie nicht noch im experimentellen Teile $z u$ besprechen sind, halten wir die folgenden für erwähnenswert ${ }^{2}$ ).

Aus den von $G$ uérin-Varry ${ }^{3}$, J. hat lder ${ }^{4}$, C. Schmidt ${ }^{5}$, Cramer ${ }^{6}$ ) lind Kirchner und B. Tollens ${ }^{7}$ mitgeteilten Analysen ist abzuleiten und heute auch wohl allgemein a) anerkannt, daB gereinigter Leinsamenschleim nach dem Trocknen bei $100^{\circ}$ die Zusammensetzung $\mathrm{C}_{6} \mathrm{H}_{10} \mathrm{O}_{5}$ besitzt. A. Hilger und $S$. Rothenfusser ${ }^{9}$ ) haben sich dem allerdings nicht angeschlossen, sondern ant gegeben, daß ihre ,Arbeiten berechtigen, für die Elementarzusammensetzung des Lsinsamenschleims die Formel aufzustellen:

$$
\left.2\left(\mathrm{C}_{6} \mathrm{H}_{10} \mathrm{O}_{5}\right) .2\left(\mathrm{C}_{5} \mathrm{H}_{8} \mathrm{O}_{4}\right)^{* 10}\right) \text {. }
$$

2) Vgl. auch B. Tolle us, "Kurzes Handbuch" der Kohlenhydrate“, 3. Aufl. (Leipzig 1914), 488. Von sonstigen Untersuchungen siehe auch noch $\$ . H$. Vines, Proc. Roy. Soc. 28, 218 (1878); A. Jorissen, Bull. Acad. Belgique [3] 5, 751 (1883); F. St o hm ann, C. von Rechenberg. H. Wilsing und P. Rodat Landw. Jahrb. 1884,549; R. W. B a ue, Jourt. prakt. Chem. [2] 30, 367 (1884) und J.Wiesner, Wiener Monatshefte 6, 592 (1885).

3) Guérin-Varry, Ann. Chim. Plys. 49, 283 (1832).

4) J. Mulder, Journ. prakt. Cliem. 15, 293 (1838). $(1844)$

5) C. Schmidt, Liebig's Ann, d. Chemie 51, 29

6) Cramer, Pflanzenphysiologische Untersuchungen $3,6$.

7) W. Kirchner und B. Tollens, Liebig's Ann. d. Chemie 175, 215 (1875).

8) Vgl. B. Toilens, loc. cit.

9) A. Hilger und S. Rothenfusser, Ber. d. Deutsch. chem. Ges. 36, 3198 (1903).

10) Analysenzahlen finden sich allerdings in dieser. Abharidlung nicht. Uebrigens hat $B$. Tollens, loc. cit.: in seinem Handbuche von dieser Mitteilung gar keine Notiz genommen. 
Die eingehenden Untersuchungen von Kirchner und Tollens ${ }^{11}$ ) lehrten, daB der gereinigte Leinsamenschleim durch 11/4 prozenige Schwefelsäure nur ziemlich schwierig hydrolysiert wird und dabei unter Abscheidung von nicht mehr als ungefähr 4 Proz. Zellulose in rechtsdrehende Gemenge von Glykose und Gummi zerfällt, und R.W. $\mathrm{B}$ a u e ${ }^{12}$ ) berichtete später, daß er bei der Einwirkung von verdünnter Schwefelsäure auf Leinsamen ebenfalls die Bildung von Dextrose beobachtet habe. Hilger und Rothenfusser ${ }^{13}$ ) dagegen teilten mit: "Durch die Hydrolyse des Schleimes mit 0,5-1 prozentiger Sctıwefelsäure entsteht nicht nur, wie bisher angenommen wurde, Dextrose, sondern Galaktose, Dextrose, Arabinose und Xylose. Bei der Hydrolyse des Schleims wurde ferner ein Nebenprodukt von saurem Charakter erhalten, dessen Bariumverbindung und auch Elementaranalyse beweisen, dab Pentane und Hexane in diesem Körper angenommen werden müssen".

Guérin-Varry 14 hat beobachtet, daß ein Teil des Schleims mit Salpetersäure Schleimsäure liefert, und diese Beobachtung ist später von Edward $C u l l i m a n ~ j u n .{ }^{15}$ ) bestätigt und von Hilger und Rothenfusser ${ }^{16}$ ) dahin erweitert worden, daß bei der Einwirkung von Salpetersäure auf den Schleim neben Schleimsäure auch noch reichlich Furfurol entstehe. Es darf aber nicht übersehen werden, daß $\mathrm{H}$. Fudakows k i ${ }^{17}$ ) bei der Verdauung von Leinsamenschleim mit künstlichem Magensaft eine teilweise kristallisierende Glykose erhalten hat, die mit Salpetersäure behandelt, keine Schleimsäure lieferte.

Was schließlich noch den Aschegehalt des Leinsamenschleims anbelangt ${ }^{18}$ ), so fanden Schmidt ${ }^{19}$ ) 0,8 Proz., Th. Anderson ${ }^{20}$ ) 3,33 Proz., S. Cloëz ${ }^{21)} 3,90$ Proz., Kirchner und Tollens ${ }^{22}$ ) 2,88 Proz., Edward

11) W. Kirchner und B. Tollens, loc. cit.

12) R. W. B a ue r, Landw. Vers.-Stat. 40, 480 (1892).

18) A. Hilger und $S$. Rothenfusser, loc, cit.

14) Guérin .Varty, loc. cit.

15) Edward Culiman. jun., Amer. Apoth. Ztg. 5, 304 (1884); Chem. Zentrablatt 1884, 744.

16) A. Hilger und S. Rothenfusser, loc. cit.

17) H. Fuda kow ski, Ber. d. Deutsch. chem. Ges. 9, $1602(1876) ; 11,1069(1878)$.

18) Außer den im Texte zitierten Untersuchungen vgl. noch besonders die Mitteilungen von E. Marchand, Am. Chim. Phys. [4] 8, 320 (1866).

19) C. Schmidt, loc. cit.

20) Th. Anderson, Joum. of the Agric. Soc. of the Hight: Soc., new series, No. 69, 376 (1860).

21) S. Clo $2 z$, Compt. rend. 61, 236, 321 und 981 (1866); Bull: soc. chim. [2] 3, 41 und 50 (1866).

22) W. Kirchner und B. Tollens, loc. cit.
Culliman jun. ${ }^{23}$ ) ungefähr 10 Proz., Al. Her$\left.z^{\circ} \operatorname{gg}^{24}\right) 3.5$ Proz. und A. Hilger und Rothenfusser ${ }^{25}$ ) 0,61 Proz.. C. Ravenna und $M$. Zamorani ${ }^{26}$ ) extrahierten der Schleim aus $1 \mathrm{~kg}$ Leinsamen, verdampften die erhaltene Flüssigkeit zur Trockene und erhielter beim Verbrennen dieses Rückstands $3,042 \mathrm{~g}$ Asche, welche enthielt:

$\begin{array}{cccccccccc} & \mathrm{K} & \mathrm{Na} & \mathrm{Ca} & \mathrm{Mg} & \mathrm{Fe} & \mathrm{PO}_{4} & \mathrm{SO}_{4} & \mathrm{SiO}_{4} & \mathrm{Cl} \\ \mathrm{g} & 0,84 & 0,2 & 0,31 & 0,12 & \mathrm{Spur} & 0,046 & 0,19 & 0,12 & 0,026\end{array}$

\section{Experimenteller Teil.}

1. Herstellung der Schleimlösungen ${ }^{27}$ ).

Da der Leinsamenschleim, wenn auch nicht zu kolloidchemischen Zwecken, so doch zu den oben erwähnten Untersuchungen schon mehrfach und nach verschiedenen Verfahren hergestellt worden ist, haben wir aus der älteren Literatur folgendes zu erwähnen.

C. S ch mid t ${ }^{28}$ ) beschreibt die Bereitung der von ihm benutzten Schleimlösung folgendermaBen: "Durch Uebergießen der Saamen mit kaltem destilliertem Wasser, gelindes Umschüt. teln und Stehenlassen, Filtriten durch Flanell, Aufkochen, abermaliges Filtriren durch Flanell, Eindampfen im Wasserbade, bis zugesetzter Alkohol den Schleim in den charakteristisch zähen Fäden coagulirte, wobei ein Theil Schleim nebst allem Pflanzencasein in der alkoholischen Lösung blieb, scharfes Auspressen, Aufquellen mit etwas Wasser, Schütteln mit chlorwasserstoffhaltigem Alkohol, und mehrmalige Wiederholung dieser Operation, bis alle Kalksalze entfernt zu seyn schienen, wurde der reine Schleim dargestellt."

Kirchner und Tollens ${ }^{29}$ gingen von ausgesuchten, von schlechten Körnem usw. völlig befreiten Leinsamen aus und digerierten diese 24 Stunden lang mit dem dreifachen Gewichte Wasser. Der zähe, klare Schlein wurde durch ein Haarsieb abgetrennt, in einer Porzellanschale zum Kocben erhitzt und, so lange er heif und

23) Edward Culliman iun., loc. cit.

24) Al. Herzog. Publ. Vers.-Stat. f. Flachsbau und Flachsb. Trautenau Nr. 3; Biedermann's Centralblatt 28, $544(1899)$.

25) A. Hilger und S. Rothenfusser, loc. cit.

26) C. Ravenna und M. Zamorani, Atti R. Accad. dei Lincei Rona [5] 19, 11, 247 (1910).

27) Wir verdankten das garantictt reine Prăpatat von Leinsamen, mit dem wit arbeiten konnten, wiederum dem liebenswurdigen Entgegeniommen des Herm Dr. K. Ha as in Camistatt.

28) C. Schmidt, loc cit.

ag) W. Kirchner und B. Tollens, loc. cit. 
dünnflüssig war, durch dichtes Leinen filtriert, was gut von statten ging. Dann wurde der Schleim auf $1 / 3$ abgedampft und nach dem Erkalten mit Salzsåure bis zu stark saurer Reaktion und mit so viel starkem Alkohol versetzt, daB der Schleim gefällt war ${ }^{30}$ ). Da der Schleim nach der Entfernung des Alkohols auf Zusatz von Wasser völlig wieder aufquoll, wurde er immer wieder in Wasser verteilt und durch wiederholte Fällung mit Säure und Alkohol weiter gereinigt.

Edward Culliman jun. ${ }^{31}$ gewann den Schleim aus den Testis durch Auskochen mit Wasser oder Ausschütteln mit angesäuertem Wasser, Filtrieren, Erhitzen bis zur Koagulation des Albumins, Konzentrieren und Fällen mit Alkohol und beobachtet, daB die so hergestellte Substanz weniger durchsichtig und brüchig war als gewöhnlicher Gummi.

Hilger und Rothenfusseri2) endlich berichten folgendes: „Bei der Darstellung des Schleimkörpers . . . . wurde der Samen des Leines mit kaltem Wasser 24 Stunden lang stehen gelassen und dann die Abscheidung des Schleims durch Eingielen in 96 prozentigen Alkohol erreicht. Der Rohschleim, welcher noch reich an Mirieralbestandtheilen war, in welchen Calcium, Magnesium, Kalium, Eisen, Phosphorsäure, Kohlensäure und Spuren von Schwefelsäure nachgewiesen werden konnten, wurde einer gründlichen Reinigung mittels Anwendung von verdünnter. Salzsäure unterzogen, welche wieder aus dem Schleim durch Behandlung mit Alkohol, auch Aether in entsprechender Form vollkommen beseitigt wurde. Es gelang auf diese Weise, einen Schleim herzustellen, welcher vollkommen loslich in Wasser war...".

Wir selbst haben mit Leinsamenschleimlösungen gearbeitet, die auf folgende Weise bereitet worden waren:

1. Verfahren. - Man wusch $100 \mathrm{~g}$ Leinsamen dreimal nacheinander mit je einem Liter gekühltem Wasser auf einem Porzellansiebe gründlich $a b$, und ließ sie hierauf mit einem Liter mit Chloroform gesättigtem Wasser bei Zimmertemperatur in inniger Berülurung, indem man das Reaktionsgemisch 24 Stunden lang ent-

30) Hier mag kurz daraut hingewiesen werden, dab J. Po h 1, Zeitschr. f. physiol. Chem. 14, 151 (1889) später die Fälibarkeit von Leinsamenschleim durch Ammoniumsulfat beobachtet hat.

a1) Edward $C_{u l l i m a n}$ jun, loc. cit.

32) A. Hilget und S. Rothenfusser, loc. cit. weder auf der Schüttelmaschine schüttelte oder mechanisch rührte. Man filtrierte schlieblich unter Anwendung der von A. Gutbier, J. Huber and Z. Krivoss ${ }^{33}$ ) beschriebenen Filtriervorrichtung durch Flanell und Glaswolle $a b$, um nun das Filtrat, dessen Trackenrückstand beim Verbrennen 14,01 Proz. Asche lieferte, sogleich durch Dialyse $z u$ reinigen.

II. Verfahren. - Das Material wurde zunächst nach der 1. Methode gewaschen und extrahiert, dann aber wurde das erhaltene Filtrat mit der doppeiten Menge absolutem Alkohol vermischt. Man gewann auf diese Weise einen weißen, flockigen Niederschlag, den man auf Leinen mit Alkohol nachwusch, abtropfen ließ und sorgsam abpreBte, und der sich in kaltem, mit Chloroform gesättigtem Wasser schnell und vollständig wieder zu einer ziemlich klaren kolloiden Lösung zerteilen tieb. Das flüssige System lieferte bein Eindunsten auf den Wasserbade und Veraschung des Trockenrückstandes 6,89 Proz. Asche und wurde ebenfalls immer sogleich der Dialyse unterworfen.

III. Verfahren. - Man stellte zuerst unter Anwendung des zweiten Verfahrens festen Extraktstoff her, behandelte inn aber dann genau so, wie es Kirchner und Tollens ${ }^{34}$ ) angegeben haben. Das auf diese Weise durch wiederholie Failung mit Salzsäure und absolutem Alkohol gereinigte Präparat lieferte bei der Behandlung mit Chloroformwasser bei Zimmertemperatur flüssige Systeme, deren Aschegehalt zu durchschnittlich 4,9 Proz. ermittelt wurde. Auch diese Schleimlösungen wurden dialysiert.

\section{Verhalten bei der Dialyse.}

Ueber das Verhalten der flüssigen Systeme, die im folgenden je nach den Methoden, nach welchen sie bereitet wurden, als System I, II und III bezeichnet werden sollen, bei der Dialyse geben die in Tabelle $1-$ III und in Fig $1-3$ zusammengestellten Versuchsergebrisse Aufschluf. Man nahm während des Reinigungsprozesses täglich Proben der Flüssigkeit heraus und bestimmte an thnen den Aschegehalt des durch Eindunsten auf dom Wasserbade gewonnenen Trockentuckstands mit den folgenden Resultaten:

33) A. Gutbier, J. Huber und Z. Krivoss, Koll.-Zeitschr. 18, 129 (1915).

34) W. Kirchner und B. Tollens, loc: cit 
Tabelle!.

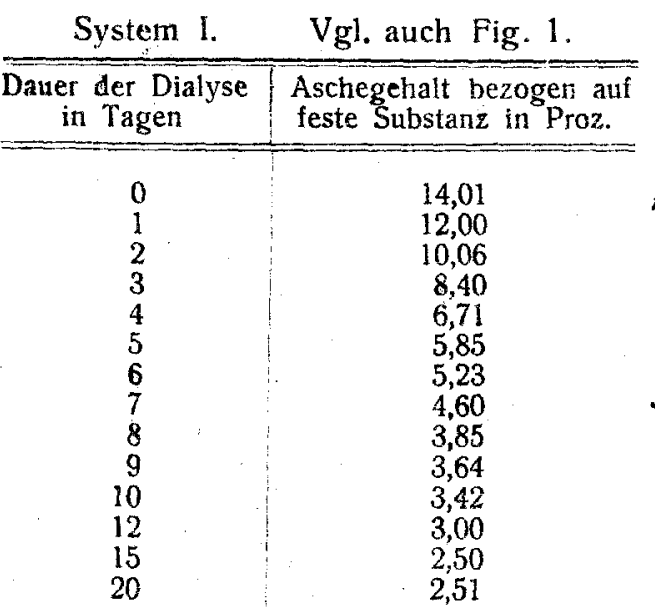

Nach beendeter Dialyse lag eine ziemlich : klare, schwach gelbliche kolloide Lösung vor.

$$
\text { Tabelle II. }
$$

System II. Vgl. auch Fig. 2.

\begin{tabular}{c|c}
\hline $\begin{array}{c}\text { Dauer der Diaiyse } \\
\text { in Tagen }\end{array}$ & $\begin{array}{c}\text { Aschegehalt bezogen auf } \\
\text { feste Substanz in Proz. }\end{array}$ \\
\hline 0 & 6,89 \\
1 & 5,33 \\
2 & 4,29 \\
3 & 3,58 \\
4 & 3,00 \\
5 & 2,41 \\
6 & 2,20 \\
7 & 2,19 \\
12 & 2,0 \\
17 & 2,0
\end{tabular}

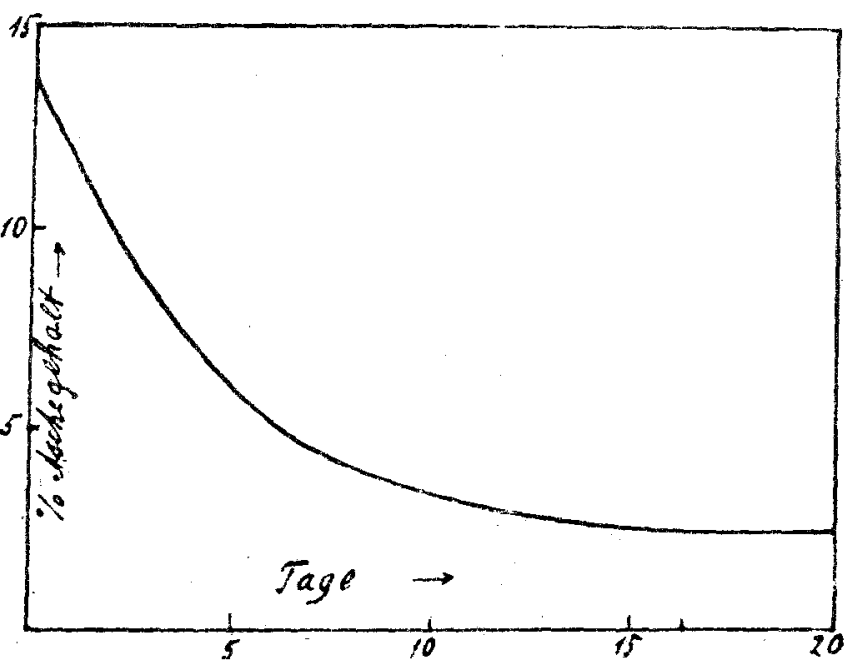

Fig. 1

Als die Flüssigkeit aus dem Pergamentschlauche herausgegossen wurde, erwies sie sich vollkommen klar und farblos.

Tabelle III.

System III.

\begin{tabular}{c|c}
\hline $\begin{array}{c}\text { Dauer der Dialyse } \\
\text { in Tagen }\end{array}$ & $\begin{array}{c}\text { Aschegehalt bezogen auf } \\
\text { feste Substanz in Proz. }\end{array}$ \\
\hline 0 & 4,9 \\
1 & 4,0 \\
2 & 3,5 \\
3 & 2,14 \\
4 & 2,11 \\
5 & 2,10 \\
8 & 2,10
\end{tabular}

Auch hier lag nach Beendigung der Dialyse eine vollkommen klare und farblose kolloide Lösung vor.

Aus den Bestimmungen selbst folgt, daf der Reinigungsprozeb bei den drei Systemen immer

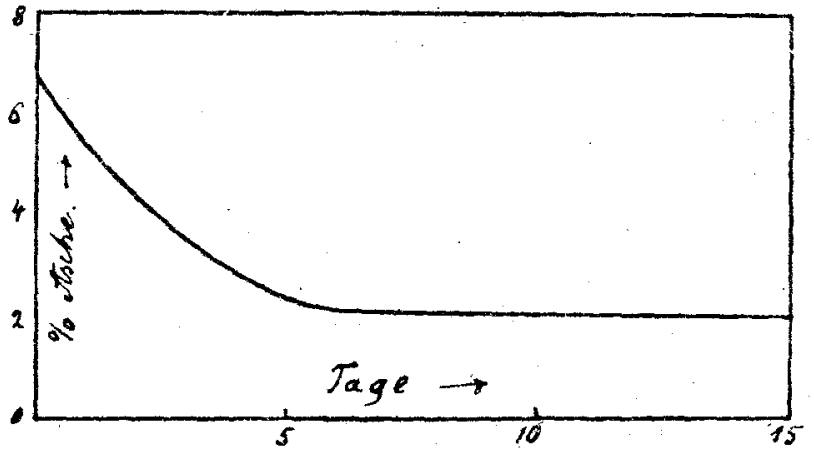

Fig. 2

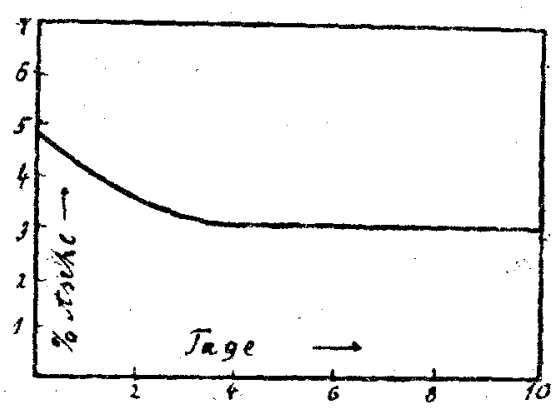

Fig. 3

in gewünschter Weise mit gutem Erfolge vor sich ging.

\section{Alterangserscheinungen.}

Wir haben uns wiederum zunächst mit den für kolloidchemische Zwecke so wichtigen Alterungserscheinungen beschäftigt, die Leinsamenschleimlösungen aufweisen, und können auf Grund zahlreicher Versuchsreihen unsere Erfahrungen folgendermaßen zusammeniassen: 
System 1. - Nach dem I. Verfahren hergestellte Flüssigkeiten halten sich allgemein nicht in zufriedenstellender Weise. Verwendet man als Untersuchungsobjekt $z$. B. eine mit reinem Wasser frisch bereitere 0,32 prozentige kolloide Lösung, so ist diese zunächst wohl ziemlich klar, weist aber schon nach 4-5 Tagen eine schwache Trübung auf und sondert im Verlaufe der zweiten Woche Schlieren ab, die sich nach und nach vermehren, sich durch Umschütteln der Flüssigkeit wohl wieder zerteilen lassen, dann aber sich aus dem trüb gewordenen Systeme sehr schnell wieder bilden. Wird eine mit reinem Wasser angesetzte 0,16 prozentige Flüssigkeit stehen gelassen, so beobachtet man schon am ersten Tage eine Trübung, am zweiten Tage Bildung von Schlieren und nach 5 -6 Tagen das Auftreten von Pilzkulturen, die das System schnell zerstören. Eine ebenfalls mit reinem Wasser hergestellte 0,08 prozentige Versuchslösung trübt sich wiederum schon am ersten Tage, läßt nach 48 Stunden starke Schlieren crkennen und weist nach fünf Tagen starke Pilzbildung auf.

Etwas besser werden die Resultate, wenn man an Stelle des reinen Wassers mit Chloroform gesättigtes Wasser als Dispersionsmittel benutzt oder die mit reinem Wasser angesetzten Systeme mit einem geringen Ueberschusse von Chloroform schüttelt und mit diesem unterschichtet dânn stehen läßt. Die Flüssigkeiten - untersucht wurden solche bis $\mathrm{zu}$ einem Gehalte von 0,38 Proz. - - selbst bleiben unter diesen Umständen wochenlang vollständig klar und setzen naturgemäß auch nicht die geringsten Spuren von Pilzkulturen an; da $B$ aber trotzdem langsam eine Zustandsänderung der Systeme vor sich geht, beobachtet man an dem unterschichteten Chloroform, das an seine Oberfläche nach und nach die Schlieren anzieht, die sich während der Alterung bilden und bei Abwesenheit von Chloroform eben lange Zeit in den Flüssigkeiten suspendiert bleiben, wahrend sie sich unter den geänderten Versuchsbedingungen schnell absetzen, so dab man bequem klare Systeme abhebern kann.

System II. -- Die nach dem II. Verfahren bereiteten Systeme dagegen zeichnen sich schon durch größere Haltbarkeit aus. Eine 0,2 prozentige, mit reinem Wasser angesetzte kolloide Lösung z. B. konnte ohne die geringste Veränderung zwei Wochen lang aufbewahrt werden und wies erst in der Mitte der dritten Woche Spuren von Schlieren auf, die sich dann allerdings ziemlich schnell vermehrten. Die ebenso bereiteten 0,1prozentigen Flüssigkeiten wurden durch Schlierenbildung erst nach Verlauf von drei Wochen, 0,05 prozentige Systeme in der fünften Woche verändert, während 0,025 prozentige Schleimlösungen sich sieben Wochen lang unverändert aufbewahren ließen. Bei Verwendung von mit Chloroform gesattigtem Wasser als Dispersionsmittel konnten kolloide Lösungen von hervorragend großer Beständigkeit gewonnen werden.

System III. - Als in bezug auf ihre Haltbarkeit ganz ausgezeichnet haben sich bei unseren Versuchen diejenigen Systeme erwiesen, welche aus dem nach dem III. Verfahren bereiteten Extraktstoffe sich herstellen ließen. An ihnen konnten im Verlaufe einer neunwöchigen Beobachtungsdauer Zustandsänderungen irgend welcher Art nicht konstatiert werden.

In bester Uebereinstimmung mit diesen makroskopischen Beobachiungen stehen die Ergebnisse der Viskosiättsbestimmungen, von denen wir über die folgenden kurz berichten wollen.

System 1. - Eine sechs Tage lang dialysierte Flüssigkeir, die einen Gehalt an Kolloidsubstanz von 0,3 Proz. besaß, wurde bei $25^{\circ}$ mit den in Tabelle IV und in Fig. 4 verzeichneten Ergebnissen in bezug auf ihre Zähflüssigkeit gemessen.

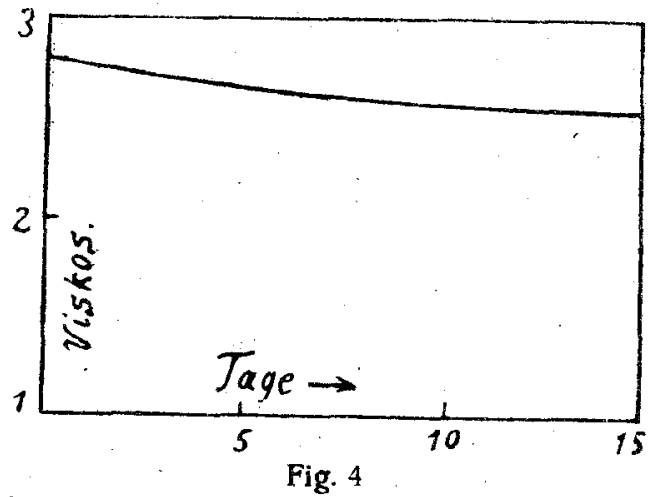

Tabelle IV.

$\begin{array}{llllllllll}\text { Tage } 0 & 1 & 2 & 3 & 4 & 6 & 7 & 9 & 12 & 14\end{array}$ $\begin{array}{lllllllllllll}\eta & 2,80 & 2,79 & 2,74 & 2,72 & 2,70 & 2,66 & 2,64 & 2,61 & 2,58 & 2,58\end{array}$

Man erkennt aus diesen Resultaten, daB die Viskosität langsam und ziemlich regelmäftig abnimmt.

Sy s t e m II. - - An einer dialysierten 0,2 prozentigen kolloiden Lösung wurde durch die in Tabelle $V$ zusammengestellten, bei $25^{\circ}$ ausgeführten Viskositätsmessungen nachgewiesen, daß, wenn auch geringe Schwankungen festgestellt wurden, die Zähigkeit der nach dem II. 
Verfahren hergestellten Systeme kaum eine Veränderung mit der Zeit erfährt.

$$
\begin{array}{ccccccccccc}
\multicolumn{10}{c}{\text { Tabelle }} & \text { V. } \\
\text { Tage } & 0 & 2 & 3 & 4 & 5 & 6 & 8 & 9 & 11 & 14 \\
\eta & 2,59 & 2,6 & 2,6 & 2,6 & 2,59 & 2,59 & 2,58 & 2,58 & 2,57 & 2,59
\end{array}
$$

System III. - Bestimmt man bei $25^{\circ}$ die relativen Viskositäten von nach dem III. Verfahren bereiteten kolloiden Lösungen, so findet man, daß innerhalb eines Zeitraums von 14 Tagen nicht die geringste Veränderung cintritt. Wir stellten $z$. B. an $0,3-, 0,2-$ und 0,1 prozentigen Systemen fest, daB die Aenderungen der Durchlaufzeiten sich innerhalb der Versuchsfehler bewegten.

\section{EinfluB der Konzentration.}

Wir stellten in verschiedenen Versuchsreihen fest, daß die relativen Viskositäțen mit steigender Konzentration an Kolloidsubstanz gleichmäßig und nach einer geraden Linie zunehmen, wie wir das ja auch schon bei anderen derartigen Extrakfflüssigkeiten beobachtet haben. $\mathrm{Da}$ vollständige Uebereinstimmung herrschte, genügt es, wenn wir in Tabelle VI und in Fig. 5 die an den nach dem I. Verfahren bereiteten kolloiden Lösungen und in Tabelle VIl und in Fig. 6 die an Systemen II bei $25^{\circ}$ erhaltenten Versuchsergebnisse als Beleg für das soeben Gesagte mitteilen.

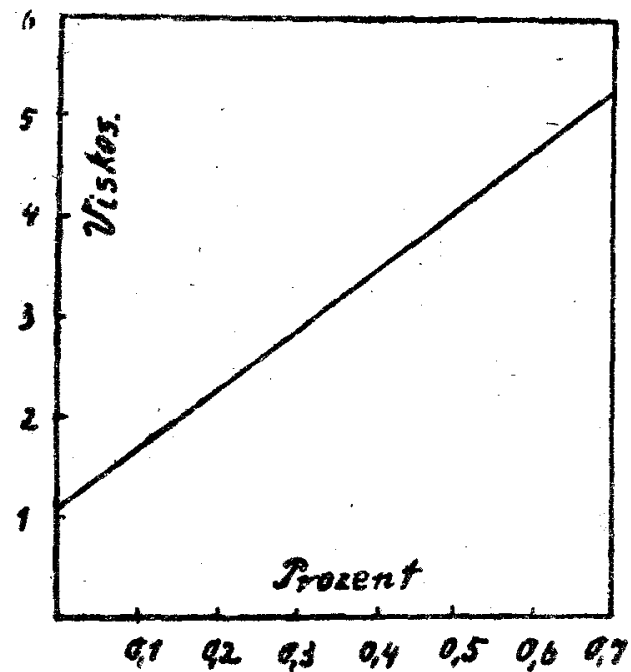

Fig. 5

Tabelle VI.

Konzenzration in Proz.

$\begin{array}{lllllll} & 0.02 & 0,04 & 0,08 & 0,17 & 0,34 & 0,68 \\ \eta & 1,09 & 1,24 & 1,48 & 2,00 & 3,06 & 5,12\end{array}$

Tabelle VII.

\begin{tabular}{cccccc}
\multicolumn{2}{c}{ Konzentration in Proz. } & & & \\
& 0,012 & 0,025 & 0,05 & 0,1 & 0,19 \\
* & 1,11 & 1,29 & 1,68 & 2,47 & $\mathbf{3 , 7 4}$
\end{tabular}

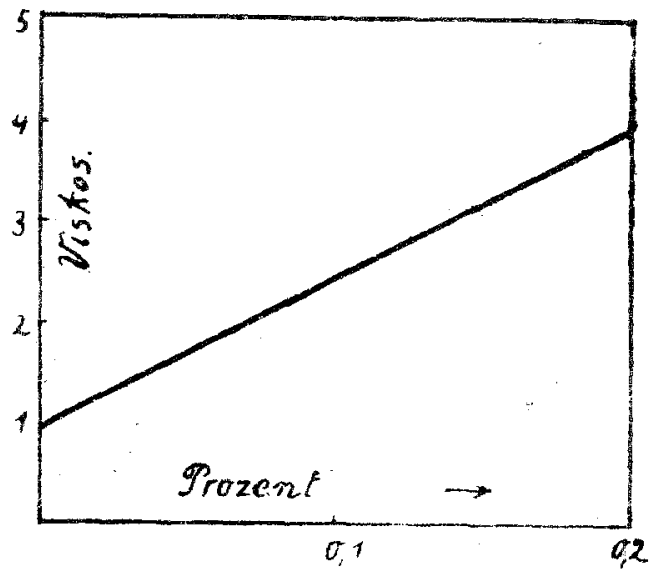

Fig. 6

5. Einflus der Temperatur.

Den Einfluß der Temperatur auf Leinsamenschleimlösungen haben wir an den nach dem 1. und nach dem II. Verfahren hergestellten kolloiden Lösungen in folgender Weise $2 u_{\text {mermit- }}^{*}$ teln versucht.

a) Man erhitzte die Plüssigkeiten in dem Ostwald'schen Kapillarviskosimeter im Glas. thermostaten langsam von $0^{\circ}-90^{\circ}$ und mal an den Proben in den geeigneten Temperatur-

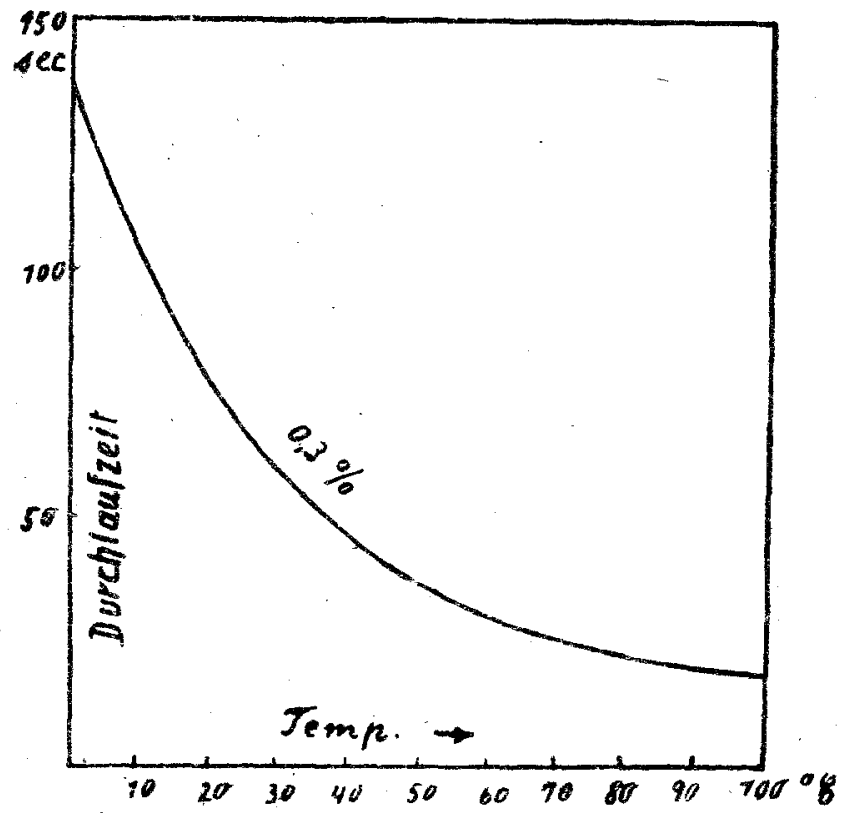

Fig. 7 
intervallen die Durchlaufzeiten, nachdem die betreffende Temperatur genau zehn Minuten lang konstant geblieben war.

Temperatur in Grad

$\begin{array}{rrrrrrrrrrr}0 & 10 & 20 & 25 & 30 & 40 & 50 & 60 & 70 & 80 & 90\end{array}$

Durchlaufzeiten in Sekunden $1364^{4} \cdot 103^{1 / 5}, 7$
Syst e m l. - Eine 0,3 prozentige Versuchsflüssigkeit lieferte so die in Tabelle VIIl und in Fig. 7 zusammengestellten Resultate.
Tabelle VIII.

$591 / 5 \quad 47^{2 / 5} \quad 373 / 5 \quad 31 \frac{1}{3} / 5 \quad 27^{2 / 5} \quad 22 \quad 201 / 5$

System 11. -... Mit einer 0,2 prozentigen kolloiden Lösung wurden die in Tabelle IX und in Fig. 8 verzeichHeten Ergebnisse gewonnen.

Die Form der Kurven entspricht vollkommen derjenigen, welche A. Gutbier, A. Iriont und E. Sa u $\mathrm{r}^{35}$ ) bei dem Extrakte von isländischem Moos, und $A$. Gutbier, Juber und $Z$. Krivoss ${ }^{36}$ ) bei dem Tragaintschleim festgestellt haben. Infolgedessen gilt das dort Gesagte auch für den Leinsamenschleim.

b) Ferner wurden die kolloiden Lösungen längere Zeit unter Rückfluß gekocht. Man nahm von Zeit zu Zeit eine Probe heraus, kühlte sie auf $25^{\circ}$ ab und bestimmte bei dieser Temperatur ihre Zähigkeit nach zehn Minuten.

Temperatur in Grad

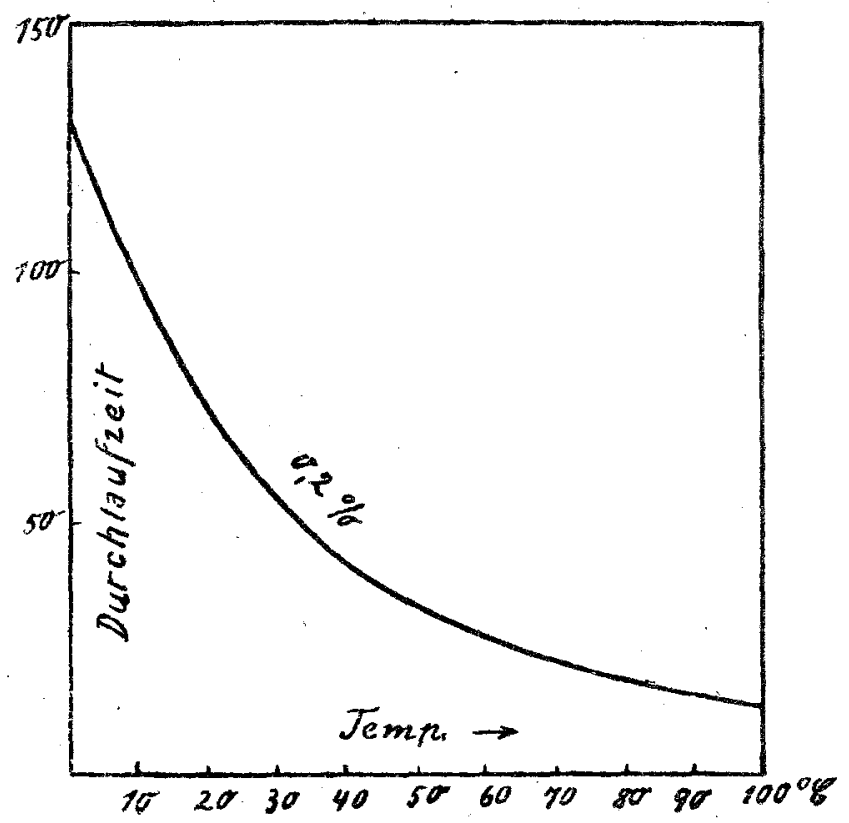

Tabelle IX.

Fig. 8

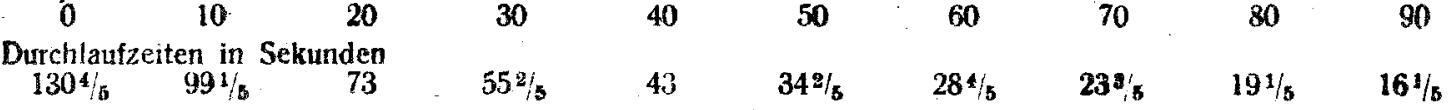

Sy stem 1 . - Eine 0,3 prozentige Versuchsflüssigkeit crgab so die in Taballe $X$ und in Fig. 9 vérzeichneten Resultate.

Die ursprünglich farblose kolloide hösung war nach zweistündigem Erhitzen gelblich nach vier Stunden gelb geworden, und schließlich lag nach neunstündigem Kochen ein intensiv gelbbraunes System vor.

35, A. Gutbier, A.Irion $\dagger$ und E. Sauer, Koll.Zeitschr. 18, $1(1916)$.

3io. A. Gutbier, J. Huber and Z. Krivoss, Koll.-Zeitschr. 18, 129 (1916).

ar) Vgl. A. Gutbier, A. Iriont u. E. Sauer. Koll.-Zeitschr. 18, 1 (1916).

Erhitzung in Stunden
Durchlaufzeiten in Sekunden
Differenz in Sekunden

Erhitzung in Stunden
Durchlaufzeiten in Sekunden
Differenz in Sekunden

Erhitzung in Stunden
Durchlaufzeiten in Sekunden
Differenz in Sekunden

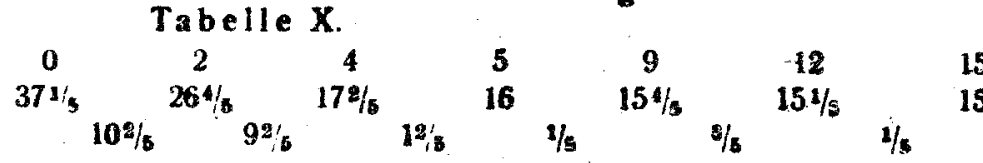

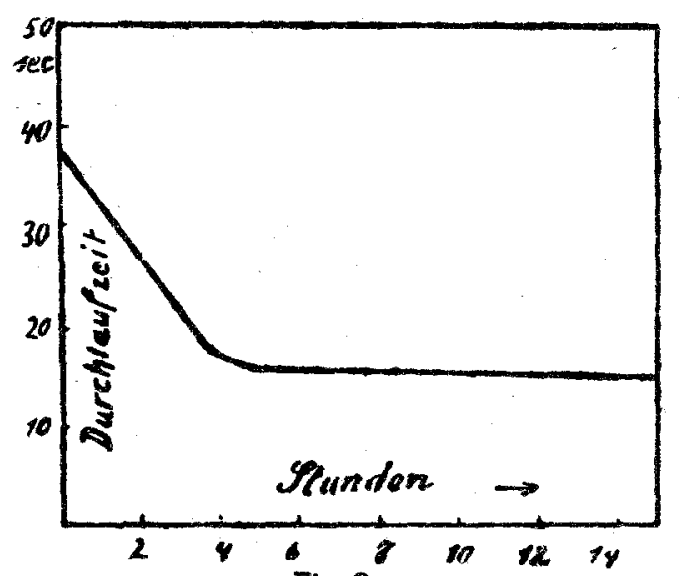

Fig. 9 
Sy s te m. II. - Bei einem 0,2 prozentigen System wurden die in Tabelle XI und in Fig. 10 zusammengestellten Ergebnisse beobachtet.

Hier färbte sich die ursprünglich farblose Versuchsflüssigkeit während des Kochens nach zwei Stunden schwach gelb, nach sieben Stunden gelb und nach zwölf Stunden gelbbraun, aber nicht so intensiv wie System 1 .

Aus diesen Messungen ergibt sich, dab die Durchlaufzeiten und damit natürlich auch die Zähigkeit der Leinsamenschleimlösungen bei längerer Zeit fortgesetzten Kochens abnehmen, um sich schlieblich nach zwölfstündigem $\mathrm{Er}$ hitzen so gut wie richt mehr zu verändern. Die Tatsache, daß zahlenmäBig ziemlich bedeutende Aenderungen der Viskosität gleichzeitig von einem deutlich erkennbaren Farbenwechsel der kolloiden. Systeme begleitet sind, weisen darauf hin, daf während des anhaltenden Erhitzens tiefgreifende Zustandsänderungen in den Leinsamenschleimlösungen vor sich gehen.

Frinitzung in Stunden Durchlaufzeiten in Sekunden Differenz in Sekunden

\section{EinfluB von Elektrolyten.}

In Anbetracht der Ergebnisse, die bei den vollständig gleichartigen diesbezüglichen Untersuchungen bei dem Extrakte von Cetraria islandica ${ }^{37}$ ) und bei den Tragantschleimlösungen ${ }^{38}$ ) festgestellt worden waren, haben wir die Einwirkung von Elektrolyten auf Leinsamenschleimlösungen in zahlreichen Versuchsreihen untersucht. Von den hier erhaltenen Resultaten wollen wir die folgenden kurz mitteilen ${ }^{39}$ ).

System I. - Wenn man nach dem I. Verfahren bereitete kolloide Lösungen verschiedener Konzentration - - untersucht wurden Flüssigkeiten mit einem Gehalte von 0,5 Proz. ab bis 0,025 Proz. - mit Salzsäure, Natronlauge oder Natriumchloridlösung verschiedener Normalität - benutzt wurden $1 / 1 \mathrm{n}$. bis $1 / 1000 \mathrm{n}$.-Lösungen in gleichen Mengenverhältnissen mischt und bei Zimmertemperatur beobachtet, lassen sich makroskopisch in den Flüssigkeitsgemischen keine Zustandsänderungen wahrnehmen, die auf einen

38) Vgl.A. Gutbier, J. Huber und Z. Krivoss, Koll.-Zeitschr. 18, 129 (1916).

39) Da sich die an System III beobachteten Erscheinungen und ausgeführten Messungen vollkommen mit denjenigen der nach dem II. Verfahren hergestellten Systeme in ihren Ergebnissen decken, verzichten wir auf eine Beschreibung der diesbezinglichen Versuche.

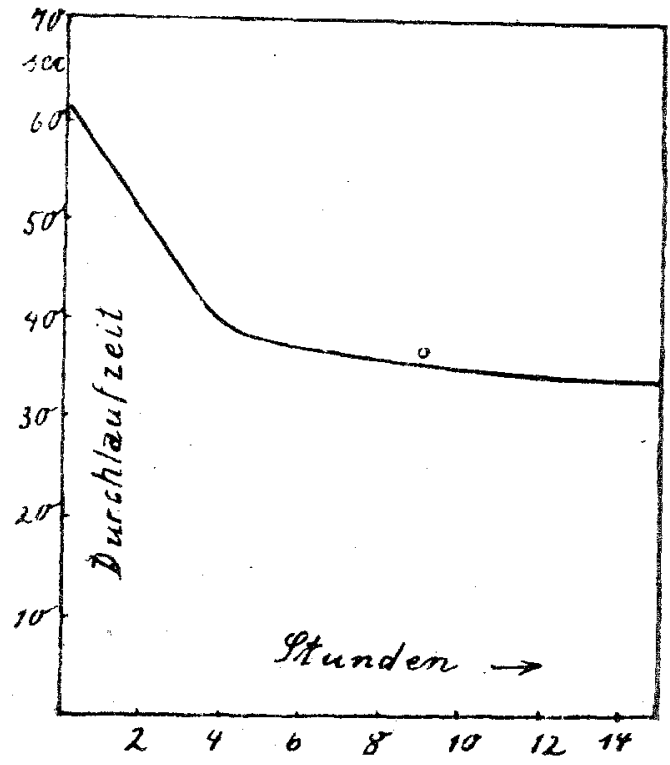

Fig. 10 
agulationsgeschwindigkeit der kolloiden Lösungen nicht erhöht, und dab ein Zusatz von Natronlauge oder von Natriumchloridlösung in den Verdünnungen. wie sie hier angewandt wurden, stabilisierend auf die Systeme einwirkt, denn unter diesen Versuchsbedingungen wird die Bildung von Schlieren in den Flüssigkeiten um einige Zeit verhindert.

$\mathrm{Zu}$ den gleichen Resultaten gelangten wir auch, als wir den Einfluß der genannten Elektrolytlösungen auf die innere Reibung der kolloiden Lösungen messend verfolgten.

Wir gingen $z$. $B$. aus von einer 0,32 prozentigen Versuchsflüssigkeit, deren Viskositătsbe- stimmungen bei $25^{\circ}$ die in Tabelle XII und in Fig. 11, I gezeichneten Ergebnisse lieferten.

Tabelle XII.

$\begin{array}{llllllllll}\text { Tage } 0 & 1 & 2 & 3 & 5 & 6 & 7 & 9 & 10 & 12\end{array}$

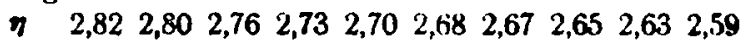

a) Einwirkung von Chlorwasserst off. - Als cin Teil dieser kolloiden Lösung mit einem Teil $\mathbf{n}$. Salzsäure vermischt und nun bei $25^{\circ}$ in gleicher Weise untersucht wurde, erhielt man die in Tabelle XIII und in Fig. !! . II zusammengestellten Resultate.

b) Einwirkung von Natriumhydro$x y d$. - In derselben Art an Mischungen von gleichen Teilen 0,32 prozentiger Leinsamenschleimlösungen und $n$. Natronlauge bei $25^{\circ}$

ausgeführte Viskositătsbestimmungen lieferten die Werte, die in Tabelle XIV und in Fig. 11 , III angegeben sind.

\begin{tabular}{|c|c|c|c|c|c|c|c|c|c|c|c|}
\hline \multicolumn{12}{|c|}{ Tabelle XIV. } \\
\hline rage & ${ }_{i, 48}$ & $1 \%$ & 1 & 2 & 3 & 5 & 6 & 7 & 8 & 10 & 11 \\
\hline$\eta$ & 1,66 & 1,63 & 1,63 & 1,62 & 1,61 & 1,60 & 1,59 & 1,58 & 1,38 & 1,57 & 1,57 \\
\hline
\end{tabular}

c) Einwirkung von Natriumchlorid. Wenn man einen Teil des kolloiden Systems mit einem Teil n.-Natriumchloridlosung vermischte und die Durchströmungsgeschwindigkelt dieser Flüssigkeit bei $25^{\circ}$ messend verfolgte, wurden die in Tabelle XV und in Fig. 11, IV verzeichneten Resultate gewonnen.

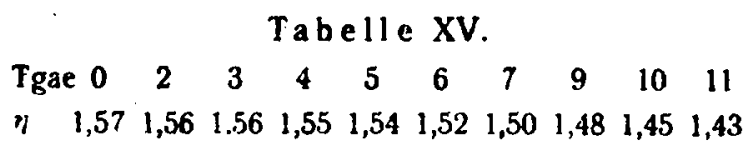

System 11. - Bei den nach dem ll. Verfahren bereiteten kolloiden Losungen wurden die gleichen Versuche mit den gleichen Ergebnissen angestellt. Auch hier übte keine der Elektrolytlösungen irgendwie schadliche Wir: kungen auf die Beständigkeit der Versuchsflüssigkeiten aus.

Im folgenden sind die Resultate einer Versuchsreihe mitgeteilt, bei welcher gleiche Teile yon 0,1 prozentiger Leinsamenschleimlosung und von Elektrolytlősungen abnehmender Normalität miteinander vermischt und bei $25^{\circ} 1 / 2$ Stunde nach ihrer Bereitung in bezug auf ihre Zähigkeit gemessen wurden.
Normalitat des $\mathrm{HCl}$

Tabelle XVI.
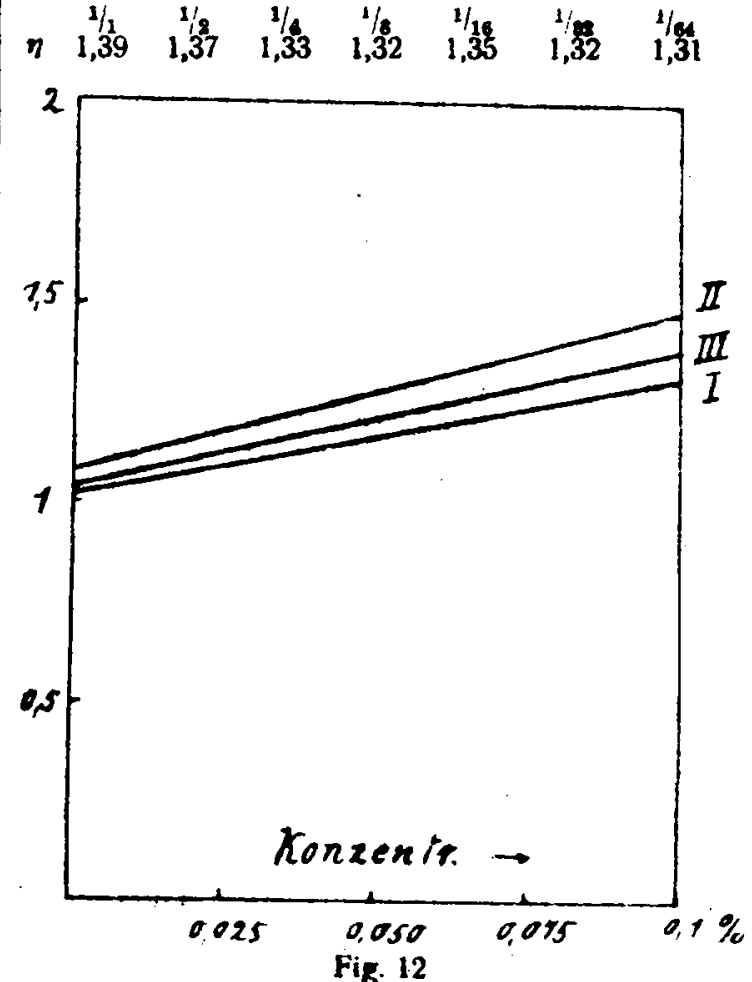
Tabelle XVII.

Normalităt des $\mathrm{NaOH}$

\begin{tabular}{|c|c|c|c|c|}
\hline $\begin{array}{c}1 / 1 \\
1,48\end{array}$ & $\begin{array}{c}1 / 8 \\
1,45\end{array}$ & $\begin{array}{l}1 / 4 \\
1,42\end{array}$ & $\begin{array}{c}1 / 6 \\
1,42\end{array}$ & $\begin{array}{r}1 / 16 \\
1,42\end{array}$ \\
\hline
\end{tabular}

Tabelle XVIII.

Normalităt des $\mathrm{NaCl}$

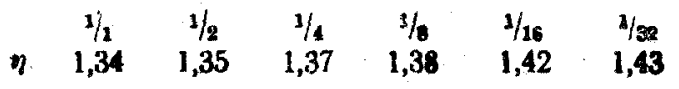

Man stellte also fest, dab die innere Reibung mit zunehmenderVerdünnung der Natriumchlorid- losung ansteigt, während sie unter dem Einflusse von Salzsäure und Natronlauge unter Jen gleichen Bedingungen sehr langsam geringer wird.

Das hat uns veranlabt, bei gleichbleibender Elektrolytkonzentrati $n$ die Konzentration der Leinsamenschleimissung in einer weiteren Versuchsreihe abnehmen $z u$ lassen und die Visknsitäten derartiger Gemische ebenfalls bei $25^{\circ}$ zu messen. Bei diesen Versuchen wurden die Resultate erhalten, die in den Tabellen XIX bis XXI und in Fig. 12 zusammengestellt sind.

$\begin{array}{ccccc}\text { Tabelle XIX. } & \text { (Vgl. Pig. 12, 1.) } & & \\ \text { Konzentration der Schleimlosung in Proz. } & 0,1 & 0,05 & 0,025 & 0,012 \\ \text { Normalität des } \mathrm{HCl} & 1 / 1 & 1 / 1 & 1 / 1 & 1 / 1 . \\ \eta & 1,31 & 1.14 & 1,09 & 1,06\end{array}$

Tahelle XX. (Vgl. Fig. 12, II.)

$\begin{array}{cccccc}\text { Konzentration der Schieimlösung in Proz. } & 0,1 & 0,05 & 0,025 & 0,012 & 0,006 \\ \text { Normalităt des NaOH } & 1 / 2 & 1 / 3 & 1 / 1 & 1 / 1 & 1 / 1 \\ \eta & 1,47 & 1,27 & 1,17 & 1,12 & 1,10\end{array}$

Tabelle XXI. (Vgl. Fig. 12, Ill.)

$\begin{array}{cccccc}\text { Konzentration der Schleimlösung in Proz. } & 0,1 & 0,05 & 0,025 & 0,012 & 0,06 \\ \text { Normalität der } \mathrm{NaCl} & 1 / \% & 1 / 2 & 1 / 1 & 1 / 1 & 1 / 1 \\ \eta & 1,38 & 1,18 & 1,10 & 1,07 & 1,05\end{array}$

Aus diesen Werten ergibt sich also bei allen drei Elektrolyten eine in normaler Weise mit der Verdännung der kolloiden Lösung sinkende Zäbigkeit.

Nach allem, was die in der vorliegenden Abhandlung beschriebenen Versuche ergeben haben, schien es aussichtsreich, den Leinsamenschleim als Schutzkolloid für anorganische Kolloide zu verwerten. Wir haben daher sogleich diesbezügliche besondere Untersuchungen ar geschlossen, über welche in den folgenden Mitteilungen Näheres berichtet werden wird.

\title{
Liter atur-Uebersicht.
}

\author{
SB. = Sitzungsbericht. $\mathrm{R} .=$ Referat.
}

1. Allgemeine Kapillarphysik

Bakker, G., Die Struktur der Kapillarschicht. (Zeitschr. f. physik. Chem. 90, 89.)

Boedeker, K. Untersuchungen über die Einwirkung periodischer Kräfte auf Tropfen, (Ann. d. Phys. [4] $46,503$.

Boutaric, A., Einige Polgerungen aus einer Formel Lord Rayleighs in bezug auf trübe Medien. (Journ. de Chim. phys. 12, 517, 1914.)

Debye, P.. Zerstreuung von Röntgenstrahlen. (Ann. d. Phys. [4] 46, 809.)

Ehrenhatt, F., Ueber kleinste Quecksilberkugeln. [Deren Mechanik, Optik und eiektrische Ladung.] (Physik. Zeitschr. 16, 227.)

Fritze, H., Die optischen Konstanten durchsichtiger Silber- und Kupferschichten. (Ann. d. Phys. [H] 47, 763.)

Ouy ot, J., Voltaeffekt und monomolekulare Schichten. (Compt. rend. 159, 307, 1914.)
Labro uste, H., Monomolekulare Schichten und Ueberschnelzung. (Compt. rend. 159, 306, 191 t.)

Lenard, P., Probleme komplexer Moleküle. I. Verdamplung und osmotischer Druck. (SB. d. Heidelberger Akad. d. Wiss., Stiftung H. Lanz; Mathem.naturw. Klasse A, 1914, 27., 28., 29 Abhandlung.) Ueber Wasserfallelektrizitat und über die Oberflächenbeschaffenheil der Flüssigkeiten. (Ann.d. Phys. [1] 47, 463.)

Meyer, E, u. W. Gerlach. Ueber die Abhängigkeit der photoelektrischen Verzögerungszeit vom Gasdruck bei Metall eilen ultramikroskopischer Größerordnung. (Ann. d. Phys [4] 47, 227.)

Pla ats, B. J. van der, Untersuchung aber $A b$. und D.spersion des Lichtes in Ferbstoffic (Ann. d. Phys. $[4]$ 47, 429.)

Pollock, J. A., Die Natur der groBen lonen sis der Luft. (Philos Mag. $[6]$ 29, 514, 1915.)

Quiricke, 0 ., Jonenwolken in feuchter expandierter Luft. (Ann. d. Phys. [1] 46, 39, 1914.) 